\title{
DEFINITION OF PRINCIPAL MATERIAL DIRECTIONS AT IRREGULAR ARTERIAL SHAPES
}

\author{
P. Bartoňová*, S. Polzer ${ }^{* *}$, J. Burša ${ }^{* * *}$
}

\begin{abstract}
For computational modelling of arterial tissues anisotropic constitutive models are preferred for which knowledge on orientation of fibres (principal material directions) is needed. In this paper the impact of different approaches to definition of principal material directions in an anisotropic model of arterial wall is evaluated. Finite element models of different regular shapes were created, as well as two idealized geometries of aortic aneurysms as examples of irregular shapes. In those geometries, difference in maximum principal stresses in the arterial wall was used for evaluation of impact of uncertainty in the principal directions. It was shown that for a cylindric shape the error is negligible but it increases for more irregular geometries. For an asymmetric aortic aneurysm the impact of different orientations of principal material directions was more than $20 \%$ which shows that the uncertainty in orientation of principal material directions may cause significant errors deteriorating completely the advantage of anisotropic material description.
\end{abstract}

Key words: arterial mechanics, constitutive model, anisotropy, principal directions

\section{Introduction}

Computational modelling and especially Finite Element Method (FEM) has become an important tool in arterial mechanics aiming at evaluation of stress-strain states in different arteries. Stresses or strains in arterial wall may be of clinical relevance at some pathologies such as aortic or brain aneurysms or atherosclerotic plaques causing arterial stenosis. In these cases credible information on stresses in arterial wall is needed for prediction of tissue rupture. While at aortic aneurysms the wall rupture endangers the patient's life by inner bleeding (in case of abdominal aneurysms the mortality rate exceeds $50 \%$ ), brain stroke can be caused by two different mechanisms. The first one is haemorrhage (intracranial bleeding) caused e.g. by rupture of a cerebral aneurysm, while local brain ischemia can be caused by stenosis or even occlusion of an artery. A stenosis increases slowly as the atherosclerotic plaque grows in the artery but it may represent itself suddenly as occlusion caused by rupture of an existing arterial plaque. The highly thrombogenic lipid core of the plaque is normally separated from the blood stream by a fibrous cap. If the fibrous cap of atheroma ruptures, the contact of blood with the lipid core causes creation of a thrombus which may occlude either the artery itself in the region of atheroma, or another branch artery downstream. The most severe consequences of this occlusion occur in the case of common or internal carotid arteries which may cause a severe brain ischemia (brain stroke). Computational modelling of carotid arteries with atheromas should enable us to distinguish the so called vulnerable plaques, i. e. plaques with high risk of rupture and consequently high risk for the patient's life.

Credible finite element modelling of stresses in arterial wall requires patient-specific (and unloaded) geometry and load of the artery, as well as sophisticated description on arterial mechanics. For arterial

\footnotetext{
Bc. Petra Bartoňová: Institute of Solid Mechanics, Mechatronics and Biomechanics, Brno University of Technology, Technická 2896/2, 61669 Brno; CZ, 160690@vutbr.cz

** Ing. Stanislav Polzer, PhD.: Department of Applied Mechanics, Technical University Ostrava; 17.listopadu 15/2172; 708 33 Ostrava; CZ,polzer@seznam.cz

*** Prof. Ing. Jiří Burša, PhD.:Institute of Solid Mechanics, Mechatronics and Biomechanics, Brno University of Technology, Technická 2896/2, 61669 Brno; CZ,bursa@fme.vutbr.cz
} 
wall, constitutive models of different levels have been proposed till now. As the structure of arterial wall, especially orientation of collagen fibres, causes its significant anisotropy, in last decades much effort has been devoted to formulation of structure based constitutive models of arterial wall and to investigation of distribution of collagen fibres in arterial walls. Most experimental results show the artery as layered structure with one (or two) dominant directions of the fibres. This structure is reflected in constitutive models formulated mostly as orthotropic with three (mutually perpendicular) principal material directions. In a tube-like (cylindric, tapering is mostly neglected) artery these principal directions correspond to circumferential, axial and radial directions of the cylinder. Especially in pathological arteries, however, their shape is far from cylindrical, the definition of principal material directions becomes ambigous and thus advantages of anisotropic constitutive models might be lost. Therefore this work is devoted to definition of principal directions at an irregular geometrical shape of an artery.

\section{Materials and methods}

The most frequently analyzed arterial pathologies are abdominal aortic aneurysms with very individual and irregular geometries, therefore also definition of principal material directions is often described in relation to them in literature. For example in (Rodriguez, 2008) and (Rodriguez, 2009), parameter $\phi$ was used as a single one for description of principal material directions of an anisotropic model. This parameter (with a constant value for the whole geometry) denotes the angle between the collagen fibers and circumferential direction of the wall. In addition, the orientation of fibers in the finite element model was described using a tangent at each integration point. Another method of determination of principal material directions is applied in (Joldes, 2016) for an anisotropic model. Although local coordinate systems should by used according to the authors, the arrangement of fibers in the global system has been chosen due to lower complexity. A specific way of definition of principal material directions is shown in Figure 1.

a

b
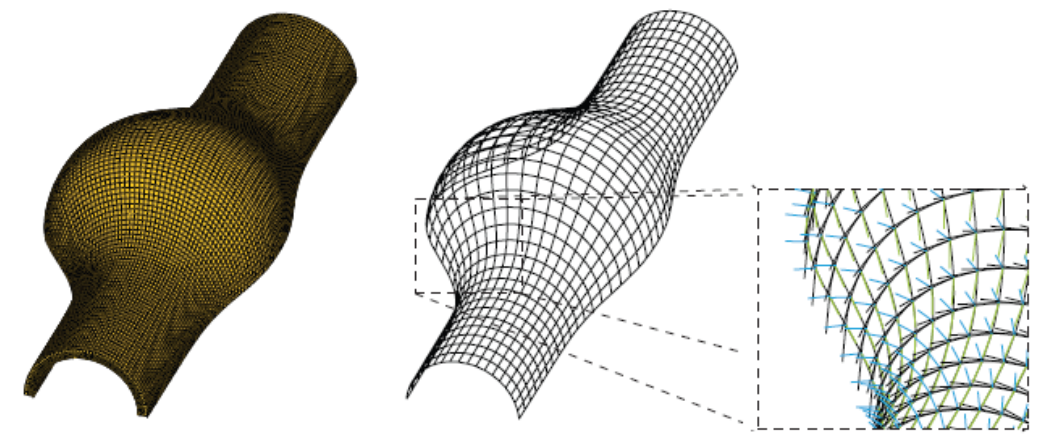

Fig. 1: In (Di Achillé, 2011), orientation of the fibers in the aneurysm wall was determined together with the mesh (part a) of the model. The model was divided by circles in parallel equidistant planes and axial equidistant spline curves. Material orientation is then defined using local coordinate systems with axial and circumferencial directions given by tangents to spline curves and circles, respectively (part b).

Another method of definition is set by (Ahamed, 2016) where local coordinates are defined by 3D polygonal centerline and its shortest connections to the centers of the elements, as shown in Figure 2. In (Vande Geest, 2008) the 3D centerline was used as the first source for local longitudinal direction. The local coordinate systems can then be determined by the normal orientation of the shell which is defined automatically together with circumferential direction computed as vector product of longitudinal and normal direction.

It is evident from the above references that principal material directions evaluated using different approaches may differ significantly but, from the practical or even clinical point of view, the impact of these uncertainties on the calculated stress field, especially on maximum principal stress, is decisive. Therefore we applied two different approaches for several idealized model geometries, ranging from a cylindrical tube to an asymmetric aortic aneurysm. All the other features of both models, except for orientation of principal material directions, were the same, including the load, boundary conditions and the constitutive model. We used the exponential anisotropic model by Holzapfel et al. (2000) 
characterized by neo-Hookean matrix and the following formula for the anisotropic part of the strain energy density function:

$$
W_{\text {aniso }\left(I_{4}, I_{6}\right)}=\frac{k_{1}}{2 k_{2}} \sum_{i=4,6}\left(e^{k_{2}\left(I_{i}-1\right)^{2}}-1\right)
$$

where $\mathrm{k}_{1}, \mathrm{k}_{2}$ are material parameters and $\mathrm{I}_{4}, \mathrm{I}_{6}$ are pseudo-invariants of Cauchy-Green deformation tensor which depend additionally on the structural tensor characterizing directions of the fibres.

We compared two approaches to definition of principal material directions. Approach A was based on cylindrical coordinate system with circumferential, axial and radial directions of the cylinder applied globally throughout all the model geometry. In approach B, the local principal directions were defined similarly to (Vande Geest, 2008) as follows:

- Radial direction - defined as normal direction to the inner arterial surface in the given point. The respective local tangential plane is the plane tangential to the surface in this point.

- Axial direction - it is represented by the line of intersection of the local tangential plane with the plane given by tangent of the axis of artery and the investigated point. The (curvilinear) axis of artery is created by centroids of its individual cross sections.

- Circumferential direction - lies in the local tangential plane and is defined as vector product of radial and axial direction.

\section{Results}

The maximum principal stresses in both models were compared in different types of geometries (see Figure 2 for examples), and the relative deviation $\Delta \sigma$ was calculated as:

$$
\Delta \sigma=\frac{\left|\sigma_{B-} \sigma_{A}\right|}{\sigma_{B}}
$$
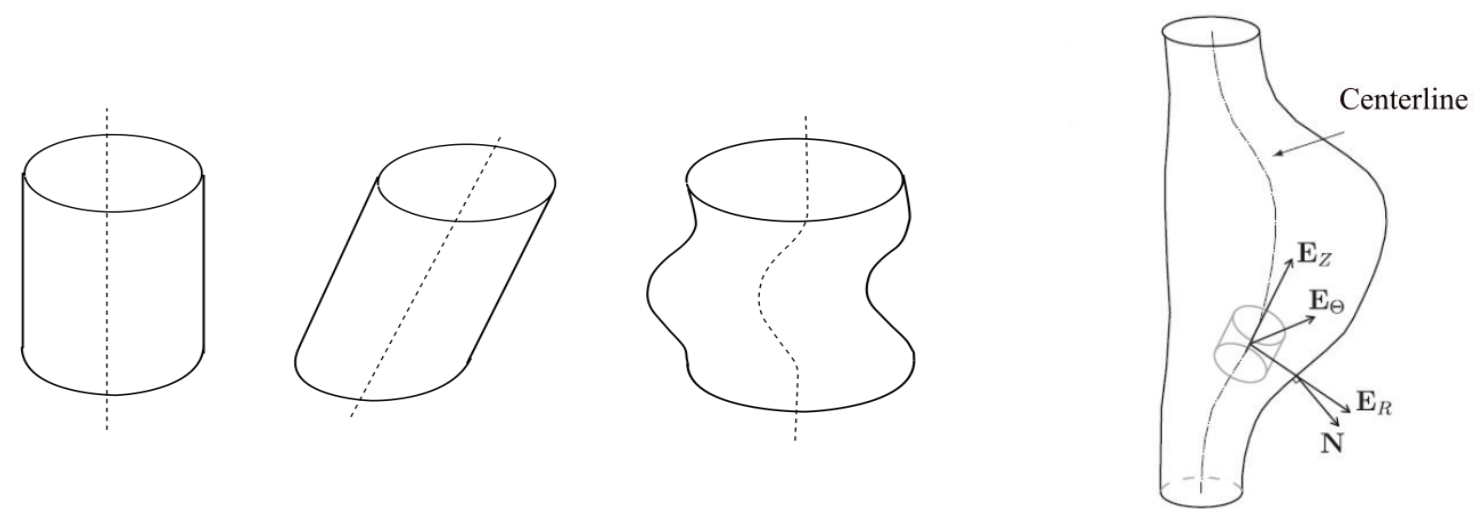

Fig. 2: Different simplified geometries of tubes used as models. From left: Cylindrical tube, sloping tube, tube with wavy centerline, asymmetric aortic abdominal aneurysm (with definition of principal material directions by approach in (Ahamed, 2016)).

The results for different simplified arterial geometries are presented in Table 1 where $\sigma_{A}$ and $\sigma_{B}$ correspond to results of both approaches.

Table 1: Maximal values of first principal stress for different simplified geometries and orientations of principal material directions

\begin{tabular}{lrrr}
\hline Geometry & $\boldsymbol{\sigma}_{\mathbf{A}}[\mathbf{k P a}]$ & $\boldsymbol{\sigma}_{\mathbf{B}}[\mathbf{k P a}]$ & $\boldsymbol{\Delta} \boldsymbol{\sigma}[\boldsymbol{\%}]$ \\
\hline Cylindrical tube & 194 & 196 & 1.17 \\
Sloping tube & 237 & 255 & 7.33 \\
Tube with wavy centerline & 264 & 247 & 7.20 \\
Symmetrical aneurysm & 341 & 313 & 9.08 \\
Asymmetric aneurysm & 277 & 347 & 20.17 \\
\hline
\end{tabular}




\section{Discussion}

The above results represent, to the best authors' knowledge, the first attempt to assess the impact of uncertainties in definition of principal material directions in arterial wall on the calculated stress. The results show that for cylindric geometry the differences correspond to the numerical error of finite element method itself. For other regular geometries the impact is higher, typically on the order of $10^{0} \%$. For asymmetric geometry of abdominal aortic aneurysm (or for an artery with atheroma being similarly asymmetric) the error exceeds $20 \%$ and becomes very significant.

\section{Conclusion}

The FE simulations have shown that impact of the way of definition of principal material directions in the arterial wall is significant in asymmetric geometries. An even higher impact can be expected for completely irregular patient-specific geometries. Thus it has been proven that for a meaningful application of anisotropic constitutive models of arterial wall the manner of determination of principal material directions is significant. Consequently for meaningful application, the same approach should be used in experimental analysis evaluating the local orientation of fibres as well as in determination of the local coordinate system of the structure in the applied anisotropic constitutive model.

\section{Acknowledgement}

This work has been supported through NETME CENTRE PLUS (LO 1202) by financial means from the Ministry of Education, Youth and Sports under the National Sustainability Programme I and by the Czech Science Foundation project no.18-13663S.

\section{References}

Ahamed, T., Dorfmann, L. and Ogden, R. W. (2016) Modelling of residually stressed materials with application to AAA, Journal of the Mechanical Behavior of Biomedical Materials, 61, 221-234.

Di Achillé, P., Celi, S., Di Puccio, F. and Forte, P. (2011) Anisotropic AAA: Computational comparison between four and two fiber family material models, Journal of Biomechanics, 44(13), 2418-2426.

Joldes, G. R., Miller, K., Wittek, A. and Doyle B. (2016) A simple, effective and clinically applicable method to compute abdominal aortic aneurysm wall stress, Journal of the Mechanical Behavior of Biomedical Materials, $58,139-148$.

Rodriguez, J. F., Ruiz, C., Doblare, M. and Holzapfel, G. A. (2008) Mechanical Stresses in Abdominal Aortic Aneurysms: Influence of Diameter, Asymmetry, and Material Anisotropy, Journal of Biomechanical Engineering, 130(2), 021023.

Rodriguez, J.F., Martufi, G., Doblaré, M. and Finol, E. A. (2009) The Effect of Material Model Formulation in the Stress Analysis of Abdominal Aortic Aneurysms, Annals of Biomedical Engineering, 37(11), 2218-2221.

Vande Geest, J. P., Schmidt, D. E., Sacks, M.S. and Vorp, D.A. (2008) The Effects of Anisotropy on the Stress Analyses of Patient-Specific Abdominal Aortic Aneurysms, Annals of Biomedical Engineering, 36(6), 921-932. 